\title{
Über die Wanderungen von Betain in Pflanzen bei einigen Vegetationsvorgängen.
}

Von

Vl. Stanĕk.

(Vorgelegt der königl. böhm. Ges. der Wissenschaften am 10. März 1911.)

(Der Redaktion zugegangen am 17. September 1911.)

In meiner letzten Arbeit über Betain (Diese Zeitschrift, Bd. 72, S. 402, 1911) habe ich festgestellt, daß diese Substanz in denjenigen Pflanzenorganen angehäuft ist, in welchen man auch die regste physiologische Tätigkeit bemerkt, in den Blättern und grünen Sprößlingen. Ich schloß daraus, daß dem Betain in dem gesamten Stickstoffwechsel der Pflanze eine bedeutende Aufgabe zuteil wird, und versuchte auch die Wanderungen von Betain bei den einzelnen Vegetationsvorgängen, besonders während des Keimens, des Wachstums, des Reifens und Absterbens der Pflanze zu verfolgen.

Bei der Bestimmung von Betain habe ich die bereits eingehend geschilderte ${ }^{1}$ ) analytische Methode angewendet.

Während meiner letzten Arbeit beobachtete ich bereits, daß die jungen Frühlingsblätter von Lycium und Atriplex canescens einen bedeutend höheren Betaingehalt aufwiesen, als die alten Herbstblätter; nun versuchte ich nachzuweisen, ob dies auch bei gleichzeitig gesammelten, jedoch verschieden alten Blättern von demselben Individuum der Fall ist.

$\mathrm{Zu}$ diesen Versuchen wählte ich die Zuckerrübe und verfolgte zugleich das Verhältnis zwischen dem gesamten Stickstoff und Betainstickstoff in Blättern und Stielen.

Die Blätter wurden am 1. September 1909 gepflückt und zwar als «äußere Blätter» die drei äußersten Reihen als *innere Blätter» die kleinen Blätter des Krautherzens, soweit

i) l. c. 
sie noch eine Trennung der Blattsubstanz vom Stiel gestatteten. Zum Stiele wurde auch die Mittelrippe genommen, während die Nebenrippen mit der Blattsubstanz zusammen verarbeitet wurden. Die Analyse ergab folgendes Ergebnis:

\begin{tabular}{|c|c|c|c|c|}
\hline & \multirow[b]{2}{*}{$\begin{array}{c}\text { Trockensubstanz } \\
\text { der frischen } \\
\text { Organe } \\
\%\end{array}$} & \multicolumn{3}{|c|}{ In der Trockensubstanz } \\
\hline & & $\begin{array}{c}\text { gesamter } \\
\text { Stickstoff } \\
\%\end{array}$ & $\begin{array}{c}\text { Betain } \\
\%\end{array}$ & $\begin{array}{c}\text { Betainstickstoff } \\
\text { vom gesamten } \\
\text { Stickstoff } \\
\%\end{array}$ \\
\hline Äußere Stiele . & 12,62 & 0,92 & 1,32 & 14,90 \\
\hline Innere Stiele . & 17,34 & 3,27 & 4,68 & 14,85 \\
\hline Äußere Blattsubstanz & 15,42 & 3,36 & 2,22 & 6,87 \\
\hline Innere Blattsubstanz & 21,98 & 5,12 & 5,10 & 10,36 \\
\hline
\end{tabular}

Der Versuch bestätigte den früheren Befund.

Die äußeren, erwachsenen Blätter hatten viel weniger Betain als die jungen, inneren Blätter, die noch unter Entwicklung standen. Auch der bereits früher beobachtete hohe Gehalt von Betain im Verhältnis zum gesamten Stickstoff in den Blattstielen hat sich gezeigt. Zur Ermittlung des Einflusses, welcher das Ableben der Blätter zur Folge hat, habe ich gemeinsam mit diesen analysierten Blättern auch die vergilbten untersucht. ${ }^{1}$ )

Ich nahm nur frische, unverwelkte Blätter von derselben Pflanze zur Untersuchung, welche von den benachbarten grünen Blättern sich nur durch ihre lichte, grünlich-gelbe Färbung unterschieden.

Es wurde wiederum die Blattsubstanz von den Stielen gesondert.

\begin{tabular}{|c|c|c|c|c|}
\hline & \multirow[b]{2}{*}{$\begin{array}{c}\text { Trocken- } \\
\text { substanz } \\
\%\end{array}$} & \multicolumn{3}{|c|}{ In der Trockensubstanz } \\
\hline & & $\begin{array}{c}\text { gesamter } \\
\text { Stickstoff } \\
\%\end{array}$ & $\begin{array}{c}\text { Betain } \\
\%\end{array}$ & $\begin{array}{c}\text { Betainstickstoff } \\
\text { vom gesamten } \\
\text { Stickstoff } \\
\%\end{array}$ \\
\hline Blattstiele . . . & 9,34 & 0,59 & 0,50 & 8,76 \\
\hline Blattsubstanz : . & 12,80 & 1,12 & 0,40 & 3,70 \\
\hline
\end{tabular}

1) Das Vergilben der Blätter ist ein Zeichen, daß die Rübenwurzeln reif werden; es zeigt sich zuerst an den äußeren Blättern und schreitet nach innen den Herzblättern zu. 
Ein Vergleich der chemischen Zusammensetzung der Blattsubstanz und der Stiele der (äußeren) grünen und gelben Blätter beweist, daß während des Ablebens die stickstoff haltigen Stoffe aus diesen Organen sich verlieren. Da der Gehalt an Betainstickstoff im Vergleich zum gesamten Stickstoff etwa auf die Hälfte sinkt, scheint es wahrscheinlich, daß das Betain von größerer Wichtigkeit für die Pflanze ist, als die anderen Stickstoffverbindungen, von welchen sie mehr in den ablebenden Blättern hinterließ.

Dieselbe Beobachtung habe ich auch bei zwei anderen Pflanzenarten gemacht, nämlich bei Lycium und Atriplex patula. Die Blätter wurden im September 1909 gesammelt, und zwar nachdem es mehrere Tage lang nicht geregnet hatte, um vor dem Einwande des Auslaugens von Betain durch das Regenwasser sicher zu sein.

Es wurden je zwei womöglich gleich große, benachbarte Blätter gepflückt, ein ganz grünes und ein gelbes, jedoch nicht verwelktes.

Die Analyse ergab:

\begin{tabular}{c|c|c|c|c|c}
\hline & Blätter & $\begin{array}{c}\text { Trocken- } \\
\text { substanz }\end{array}$ & \multicolumn{3}{|c|}{ In der Trockensubstanz } \\
\cline { 5 - 6 } & & $\begin{array}{c}\text { Stickstoff } \\
\%\end{array}$ & Betain & $\begin{array}{c}\text { Betainstickstof } \\
\text { vom gesamten } \\
\text { Stickstoff } \\
\%\end{array}$ \\
\hline Lycium & grüne & 19,60 & 3,29 & 2,46 & 7,76 \\
barbarum & gelbe & 17,30 & 1,55 & 0,50 & 3,36 \\
\hline Atriplex & grüne & 21,80 & 4,05 & 3,38 & 8,68 \\
patula & gelbe & 20,80 & 1,89 & 1,21 & 6,60
\end{tabular}

In beiden Fällen beobachteten wir eine beträchtliche Verminderung des Betaingehaltes in der Trockensubstanz der gelben Blätter und ein Abnehmen des Betainstickstoffs im Verhältnis zum gesamten Stickstoff.

Dieselbe Erscheinung wie bei dem Ableben der Blätter zeigt sich auch bei dem Reifen der Früchte, was an den Früchten von Lycium festgestellt wurde.

Da diese Pflanze gleichzeitig reife und unreife Früchte 
trägt, konnten beide an demselben Strauche gesammelt werden. Die Samen ließen sich nicht vom Fruchtfleisch quantitativ absondern, deswegen wurden die Früchte im ganzen analysiert.

\begin{tabular}{|c|c|c|c|c|}
\hline \multirow[b]{2}{*}{ Früchte } & \multirow[b]{2}{*}{$\begin{array}{l}\text { Trockensubstanz } \\
\text { der } \\
\text { frischen Früchte } \\
\%\end{array}$} & \multicolumn{3}{|c|}{ In der Trockensubstanz } \\
\hline & & $\begin{array}{c}\text { Stickstoff } \\
\%\end{array}$ & $\begin{array}{c}\text { Betain } \\
\%\end{array}$ & $\begin{array}{c}\text { Betainstickstoff } \\
\text { vom gesamten } \\
\text { Stickstoff } \\
\%\end{array}$ \\
\hline Grün & 25,00 & 3,92 & 2,43 & 6,44 \\
\hline Reif & 19,03 & 3,78 & 1,89 & 5,17 \\
\hline
\end{tabular}

Auch in den Stengeln der Samenrüben findet eine $A b-$ nahme von Betain nach Beendigung der vegetativen Tätigkeit statt. Die Trockensubstanz eines Rübenstengels während der Blütezeit im August hatte 1,42\% Betain; nachdem die Samen reif geworden, am Ende September, nur noch 0,27\%.

Alle diese Tatsachen beweisen, daß nach Beendigung der vegetativen Tätigkeit das Betain aus den reifen Organen verschwindet und wahrscheinlich in die Mutterpflanze zurückwandert. Es scheint nicht annehmbar, daß das Betain während des Reifens zersetzt wird, da in den angeführten Organen keine Spur von Trimethylamin (durch Destillation mit Magnesia) nachgewiesen werden konnte, welches stets als das erste Spaltungsprodukt von Betain erscheint. Meine weiteren Versuche betreffen die Ermittlung der quantitativen Veränderungen des Betaingehaltes während des Keimens, sowie die Frage, ob das Betain bereits während des Keimens sich bildet, und da die Anhäufung von Betain in den grünen Pflanzenorganen(Blättern und Stengeln) auffallend erscheint, versuchte ich zugleich den Einfluß des Lichtes festzustellen, welches bekanntlich die Bildung des Blattgrünes bedingt.

\section{Versuche mit Gerste.}

Das Korn hatte 85\% Trockensubstanz und 0,05\% Betain. Im April wurden in zwei Blechschalen, welche mit Ackererde auf etwa $3 \mathrm{~cm}$ hoch gefüllt waren, je $100 \mathrm{~g}$ Korn, entsprechend $0,05 \mathrm{~g}$ Betain, gesät. Eine Schale wurde mit Glas bedeckt, die andere mit einer für das Licht undurchdringlichen Platte. 
Nach 20 Tagen wurden die jungen Pflanzen oberhalb der Erde abgeschnitten und (ohne Wurzel) analysiert.

\begin{tabular}{|c|c|c|c|c|c|c|c|}
\hline \multirow{3}{*}{ Pflanzen } & \multirow{3}{*}{$\begin{array}{l}\text { Gewicht } \\
\text { der } \\
\text { frischen } \\
\text { Substanz } \\
\text { g }\end{array}$} & \multirow{3}{*}{$\begin{array}{l}\text { Gewicht } \\
\text { der } \\
\text { Trocken- } \\
\text { substanz } \\
\text { g }\end{array}$} & \multicolumn{5}{|c|}{ In der Trockensubstanz } \\
\hline & & & \multicolumn{2}{|c|}{ Stickstoff } & \multicolumn{2}{|c|}{ Betain } & \multirow{2}{*}{$\begin{array}{c}\text { Betain- } \\
\text { stickstoff vom } \\
\text { gesamten } \\
\text { Stickstoff } \\
\%\end{array}$} \\
\hline & & & $\%$ & $\mathrm{~g}$ & $\%$ & g & \\
\hline Grün & 267,0 & 15,5 & 4,26 & 0,66 & 0,64 & 0,099 & 1,64 \\
\hline Etiolisiert & 250,0 & 16,0 & 4,06 & $0,6 \check{5}$ & 0,62 & 0,099 & 1,58 \\
\hline
\end{tabular}

Es zeigte sich fast kein Unterschied in der Zusammensetzung der Trockensubstanz der Pflanzen und die Etiolisation scheint ohne Einfluß zu sein. Die gesamte Menge von Betain erhöhte sich beträchtlich; die oberirdischen ${ }^{1}$ ) Organe selbst enthielten schon zweimal so viel davon, als in dem Korn eingesät wurde.

\section{Versuch mit Rübensamen.}

Betain wurde in jungen keimenden Pflanzen (3-6 Tage alt) bestimmt, welche bei der Ermittelung der Keimfähigkeit aus den Samenknäueln ausgezogen worden waren. ${ }^{2}$ )

Die Trockensubstanz der Samen mit einem Keim von etwa $10 \mathrm{~mm}$ Länge enthielt:

$$
\begin{aligned}
& 5,01 \% \text { Stickstoff, } \\
& 0,61 \% \text { Betain. }
\end{aligned}
$$

Die Samen selbst enthielten vor dem Keimen in der Trockensubstanz

$$
\begin{aligned}
& 3,47 \% \text { Stickstoff, } \\
& 0,18 \% \text { Betain. }
\end{aligned}
$$

1) Nach dem Abschneiden der jungen Pflanzen wollte ich noch eine zweite Ernte züchten und sodann auch die Wurzeln analysieren. Dieser Versuch mißlang, indem die Vegetation von Schimmelpilzen betroffen wurde.

2) Etwa $15 \mathrm{~g}$ dieser (getrockneten) Keimlinge erhielt ich von H. E. Vitek, Vorstand der Kontrollstation der Samenzüchter, dem ich meinen besten Dank sage. 
Auch in diesem Falle stieg während der kurzen Vegetationsperiode die Menge von Betain sehr beträchtlich. Es ist jedoch nicht ausgeschlossen, daß hier das Betain der Samenhülle, welche etwa 1,5\% davon in der Trockensubstanz enthält, direkt assimiliert wurde. Da die ausgeschälten Samen meistens mechanisch beschädigt werden, habe ich zu weiteren Versuchen die Samen von anderen betainreichen Pflanzen gewählt.

Versuche mit Samen von Amaranthus caudatus.

a) Zweimal je $20 \mathrm{~g}$ von reinen, ausgeschälten Samen mit $0,13 \%$ Betain $(=0,026 \mathrm{~g})$ wurden auf porösen Tellern gesät, welche in Schalen mit Wasser hingestellt wurden. Der eine wurde mit einer Glasglocke bedeckt, der andere mit einer Blechglocke.

Nach 8 Tagen, nachdem die Pflanzen schon zwei rotgefärbte Blätter zeigten, wurden sie samt Wurzeln getrocknet und in der Trockensubstanz das Betain bestimmt. Die belichteten Pflanzen hatten $0,06 \mathrm{~g}$ Betain $(0,30 \%$ der verwendeten Samen) entsprechend einer Zunahme von $0,034 \mathrm{~g}$ oder $0,17 \%$. Bei den etiolisierten Pflanzen fand ich $0,036 \mathrm{~g}$ Betain $(=0,18 \%$ ), was einer Zunahme von $0,010 \mathrm{~g}$ oder $0,07 \%$ entspricht.

b) Bei diesem Versuche wurde unter sonst gleichen Bedingungen eine Vegetationsperiode von 12 Tagen eingehalten und die Pflanzen hatten schon deutlich grüne Blätter. Länger konnte ich nicht warten, da die etiolisierten Pflanzen anfingen schwarz zu werden. Die Analyse ergab:

in den belichteten Pflanzen 0,066 $\mathrm{g}$ Betain $=0,33 \%$, » etiolisierten $\gg 0,034 »>=0,17 \%$.

Auch in diesem Falle stieg die Menge von Betain während der ersten Vegetationsperiode, und es konnte auch ein gewisser Einfluß der Belichtung wahrgenommen werden. Da die Möglichkeit einer Aufnahme von Betain von außen her, wie bei den Rübensamen, ausgeschlossen war, kann man folgern, daß die Pflanze es selbst gebildet hat, und folglich es auch schon in den ersten Vegetationstagen bedarf. 
Die Frage, ob das Betain aus anderen stickstoff haltigen Substanzen des Samens oder durch Assimilation des Stickstoffs der Umgebung entstand, wozu einige Milligramm des Stickstoffs, welcher auch der oft ammoniakhaltigen Laboratoriumsluft entstammen könnte, genügten - kann ich in Anbetracht der minimalen Menge nicht entscheiden. Es wird notwendig, den Versuch unter zweckmäßigeren Bedingungen und mit einem mehr geeigneten Material $\mathrm{zu}$ wiederholen, ${ }^{1}$ ) wobei auch die anderen Formen des Stickstoffs, insbesondere die Proteine berücksichtigt werden. Auch scheint es mir von Wichtigkeit, festzustellen, ob die Proteine der betainhaltigen Pflanzen durch Oxydation oder Hydrolyse das Betain liefern.

\section{Versuche mit Zuckerrübenwurzeln.}

6 Rübenwurzeln, im ganzen $4,450 \mathrm{~g}$, wurden auf der Reibe zerkleinert und der Rübenbrei analysiert.

Gefunden: 19,2\%Zucker, 27,2\% Trockensubstanz. In der Trockensubstanz 1,26\% Stickstoff und 0,81\% Betain. Zweimal je 6 Rübenwurzeln von derselben Probe, von ähnlicher Form und ähnlichem Gewicht wurden in 2 Gefäße mit nassem Sand eingesetzt. Das eine Gefäß wurde mit Glas, das andere mit Blech bedeckt. Die Rüben unter dem Glas wogen im ganzen 4,300 g, die andere Partie 4,500 g. Der Versuch fing Mitte März 1910 an. Nach einer 30 tägigen Vegetationsperiode wurden die Blätter dicht am Rübenkopfe abgeschnitten, und die Wurzeln zu weiteren Versuchen im Sande belassen. Die geernteten Blätter wurden gewogen, getrocknet und analysiert.

1) R. Engel and (Diese Zeitschr., Bd, 67, S. 403) und E. Schulze und G. Trier (Diese Zeitschr., Bd. 67, S. 46) weisen auf die Möglichkeit hin, daß die Betaine aus Spaltungsprodukten der Eiweißstoffe sich bilden können. Als die zitierte Arbeit erschien, waren meine Versuche bereits beendet. Ich beabsichtige, weitere Versuche mit knospenden Trieben eines betainhaltigen Strauches vorzunehmen. 
Über die Wanderungen von Betain in Pflanzen.

Erste Ernte.

\begin{tabular}{l|c|c|c|c|c|c|c}
\hline \hline \multirow{2}{*}{ Blätter } & $\begin{array}{c}\text { Gewicht } \\
\text { der } \\
\text { frischen } \\
\text { Substanz } \\
\mathrm{g}\end{array}$ & $\begin{array}{c}\text { Gewicht } \\
\text { der } \\
\text { Trocken- } \\
\text { substanz } \\
\mathrm{g}\end{array}$ & $\begin{array}{c}\text { \% } \\
\text { Trocken- } \\
\text { substanz in } \\
\text { der frischen } \\
\text { Substanz }\end{array}$ & \multicolumn{2}{|c|}{ In der Trockensubstanz } \\
\cline { 4 - 8 } & \multicolumn{2}{|c|}{$\begin{array}{c}\text { Stickstoff } \\
\%\end{array}$} & \multicolumn{2}{|c}{ Betain } \\
Belichtet & 240,0 & 31,3 & 13,05 & 5,32 & 1,66 & 7,77 & 2,43 \\
Etiolisiert & 198,0 & 18,5 & 9,05 & 5,15 & 0,49 & 7,16 & 0,95
\end{tabular}

Nach weiteren 30 Tagen erfolgte die zweite Ernte.

\begin{tabular}{l|c|c|c|c|c|c|c}
\hline \multirow{3}{*}{ Blätter } & $\begin{array}{c}\text { Gewicht } \\
\text { der } \\
\text { frischen } \\
\text { Substanz } \\
\mathrm{g}\end{array}$ & $\begin{array}{c}\text { Gewicht } \\
\text { der } \\
\text { Trocken- } \\
\text { substanz } \\
\mathrm{g}\end{array}$ & $\begin{array}{c}\text { \% } \\
\text { Trocken- } \\
\text { substanz in } \\
\text { der frischen } \\
\text { Substanz }\end{array}$ & \multicolumn{2}{|c|}{ In der Trockensubstanz } \\
\cline { 5 - 8 } & $\begin{array}{c}\text { Stickstoff } \\
\text { Belichtet }\end{array}$ & 147,0 & 17,8 & 12,1 & \multicolumn{2}{|c|}{ Betain } \\
Etiolisiert & 145,5 & 12,2 & 8,1 & 4,60 & 0,56 & 6,62 & 0,81
\end{tabular}

Nach der zweiten Ernte wurden die Wurzeln noch 20 Tage lang im Sande belassen, um noch die dritte Ernte zu erzielen. Dabei verdarben jedoch einige Wurzeln, und zwar 5 der belichteten und 1 etiolisierte.

Dritte Ernte.

\begin{tabular}{|c|c|c|c|c|c|c|c|}
\hline \multirow{3}{*}{ Blätter } & \multirow{3}{*}{\begin{tabular}{|} 
Gewicht \\
der \\
frischen \\
Substanz \\
$\mathbf{g}$ \\
\end{tabular}} & \multirow{3}{*}{$\begin{array}{l}\text { Gewicht } \\
\text { der } \\
\text { Trocken- } \\
\text { substanz } \\
\text { g. }\end{array}$} & \multirow{3}{*}{$\begin{array}{c}\% \text { der } \\
\text { Trocken- } \\
\text { substanz von } \\
\text { der frischen } \\
\text { Substanz }\end{array}$} & \multicolumn{4}{|c|}{ In der Trockensubstanz } \\
\hline & & & & \multicolumn{2}{|c|}{ Stickstoff } & \multicolumn{2}{|c|}{ Betain } \\
\hline & & & & $\%$ & g & $\% / 0$ & g \\
\hline Belichtet & 48,0 & 6,05 & 13,00 & 3,98 & 0,24 & 5,46 & 0,34 \\
\hline Etiolisiert & 148,0 & 12,60 & 8,06 & 4,60 & 0,60 & 5,50 & 0,71 \\
\hline
\end{tabular}

Nun wurden auch noch die verbliebenen gesunden Wurzeln analysiert. Ich fand:

\begin{tabular}{c|c|c|c}
\hline \multirow{2}{*}{ Wurzeln } & $\begin{array}{c}\text { \% der } \\
\text { Trockensubstanz } \\
\text { von der } \\
\text { frischenSubstanz }\end{array}$ & \multicolumn{2}{|c}{ In der Trockensubstanz } \\
\cline { 2 - 4 } & $\begin{array}{c}\text { Stickstoff } \\
\%\end{array}$ & $\begin{array}{c}\text { Betain } \\
\%\end{array}$ \\
\hline Belichtet . . . & 20,2 & 1,03 & 0,50 \\
Etiolisiert . . . & 21,0 & 0,82 & 0,45
\end{tabular}


Da ein Teil der Wurzeln verdarb, konnte die Bilanz des Stickstoffs nicht durchgeführt werden. Im ganzen großen wurde in Übereinstimmung mit den früheren Versuchen über das Keimen der Samen bestätigt, daß Betain ohne irgend welchen Zusammenhang mit der Bildung von Chlorophyll in die Blätter übergeht.

Die etwas geringere Menge von Betain in den etolisierten Blättern kann ungezwungen durch die geringere vegetative Tätigkeit erklärt werden.

Der Betaingehalt der Trockensubstanz der Rübenwurzeln war in beiden Fällen schließlich fast derselbe, zugleich jedoch im Verhältnis zum ursprünglichen Zustande der Wurzeln um 0,30-0,35 vermindert, obzwar auch die Trockensubstanz während der Vegetation um 6-7\% abnahm. Es ist klar, daß auch während des Wachsens der Rübe Betain in die Blätter wandert, wo die Pflanze es bedarf. Die Frage, ob während dieses Wachstums Betain neu gebildet wurde, oder bloß eine Wanderung des vorhandenen stattfand, konnte ich infolge des Ablebens der Wurzeln nicht entscheiden. ${ }^{1}$ )

Als Ergebnis meiner Versuche finde ich:

1. Die Trockensubstanz der jungen Blätter enthält mehr Betain als die Trockensubstanz der alten Blätter derselben Pflanze, und auch das Verhältnis zum Gesamtstickstoff stellt sich bei den ersten höher.

2. Bei dem Reifen und Ableben der Pflanzenorgane verschwindet das Betain gleichzeitig mit den anderen Stickstoffarten. Doch vermindert sich dabei zugleich das Verhältnis zwischen Betainstickstoff und gesamtem Stickstoff. Da das wahrscheinlichste Zersetzungsprodukt von Betain, das Trimethylamin, gleichzeitig nicht nachgewiesen werden konnte, ist es annehmbar, daß Betain nach Beendigung der vegetativen Tätigkeit der Organe in die Mutterpflanze zurückwandert ein Beweis, daß Betain kein Abfallprodukt des Stickstoffwechsels

1) Das Ableben der jungen Pflanzen, welches meine Versuche unangenehm betraf, erkläre ich mir durch Einwirkung der verunreinigten Laboratoriumsluft. Bekanntlich werden junge Pflanzen schon durch Spuren von Leuchtgas stark beschädigt. 
der Pflanze ist (die Vermutung von E. Schulze und E. Trier: Diese Zeitschr., Bd. 67, S. 46).

3. Während des Keimens der Samen wird Betain gebildet. Es erübrigt, zu entscheiden, ob diese Bildung in den Reservestoffen oder in dem assimilierten Stickstoff ihre Quelle hat.

4. Während des Sprossens der Rübenwurzel wird Betain in den Blättern angehäuft und verschwindet zugleich aus der Wurzel, wodurch wiederum seine Wichtigkeit bei den Vegetationsvorgängen bestätigt wird.

5. Betain wird gebildet resp. angehäuft auch ahne Wirkung des Lichtes in etiolisierten Blättern, woraus $\mathrm{zu}$ schließen ist, daß es bei der Assimilation des Kohlenstoffs keine Rolle spielt.

Die Aufgabe des Betains im Leben der Pflanze bleibt unaufgeklärt, sowie die Aufgabe der Alkaloide. Die Lösung dieser Frage wird noch weitere Studien erfordern. Vielleicht wird uns die Untersuchung der Proteine der betainhaltigen Pflanzen näher zum Ziele bringen, sowie die Erkennung der histologischen Lokalisation von Betain in den einzelnen Organen.

Mit der erstgenannten Untersuchung bin ich beschäftigt, während die zweite Aufgabe H. Mg. Pharm. E. Senft, Beirat des Landeskulturrates, übernommen hat.

Aus dem Laboratorium der Versuchsstation für Rübenzuckerindustrie in Prag. 